\title{
GPU Acceleration of Multigrid Preconditioned Conjugate Gradient Solver on Block-Structured Cartesian Grid
}

\author{
Naoyuki Onodera* \\ Center for Computational Science and \\ e-Systems, Japan Atomic Energy \\ Agency \\ Kashiwa, Chiba, Japan \\ onodera.naoyuki@jaea.go.jp \\ Susumu Yamashita \\ Nuclear Science and Engineering \\ Center, Japan Atomic Energy Agency \\ Tokai, Ibaraki, Japan \\ yamashita.susumu@jaea.go.jp
}

\author{
Yasuhiro Idomura \\ Center for Computational Science and \\ e-Systems, Japan Atomic Energy \\ Agency \\ Kashiwa, Chiba, Japan \\ yasuhiro.idomura@jaea.go.jp
}

Takashi Shimokawabe

Information Technology Center, The

University of Tokyo

Bunkyo-ku, Tokyo, Japan

shimokawabe@cc.u-tokyo.ac.jp

\author{
Yuta Hasegawa \\ Center for Computational Science and \\ e-Systems, Japan Atomic Energy \\ Agency \\ Kashiwa, Chiba, Japan \\ hasegawa.yuta@jaea.go.jp
}

Takayuki Aoki

Global Scientific Information and

Computing Center, Tokyo Institute of

Technology

Meguro-ku, Tokyo, Japan

taoki@gsic.titech.ac.jp

\begin{abstract}
We develop a multigrid preconditioned conjugate gradient (MG-CG) solver for the pressure Poisson equation in a two-phase flow CFD code JUPITER. The JUPITER code is redesigned to realize efficient CFD simulations including complex boundaries and objects based on a block-structured Cartesian grid system. The code is written in CUDA, and is tuned to achieve high performance on GPU based supercomputers. The main kernels of the MG-CG solver achieve more than $90 \%$ of the roofline performance. The MG preconditioner is constructed based on the geometric MG method with a threestage V-cycle, and a red-black SOR (RB-SOR) smoother and its variant with cache-reuse optimization (CR-SOR) are applied at each stage. The numerical experiments are conducted for two-phase flows in a fuel bundle of a nuclear reactor. Thanks to the blockstructured data format, grids inside fuel pins are removed without performance degradation, and the total number of grids is reduced to $2.26 \times 10^{9}$, which is about $70 \%$ of the original Cartesian grid. The MG-CG solvers with the RB-SOR and CR-SOR smoothers reduce the number of iterations to less than $15 \%$ and $9 \%$ of the original preconditioned CG method, leading to 3.1- and 5.9-times speedups, respectively. In the strong scaling test, the MG-CG solver with the CR-SOR smoother is accelerated by 2.1 times between 64 and 256 GPUs. The obtained performance indicates that the MG-CG solver designed for the block-structured grid is highly efficient and enables large-scale simulations of two-phase flows on GPU based supercomputers.
\end{abstract}

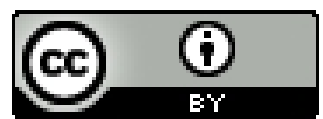

This work is licensed under a Creative Commons Attribution International 4.0 License. HPCAsia 2021, January 20-22, 2021, Virtual Event, Republic of Korea

(C) 2021 Association for Computing Machinery.

ACM ISBN 978-1-4503-8842-9/21/01 ..\$15.00

https://doi.org/10.1145/3432261.3432273

\section{CCS CONCEPTS}

- Applied computing $\rightarrow$ Physical sciences and engineering;

\section{KEYWORDS}

Multigrid method, Krylov method, GPU, Block-structured AMR, CFD simulation

\section{ACM Reference Format:}

Naoyuki Onodera, Yasuhiro Idomura, Yuta Hasegawa, Susumu Yamashita, Takashi Shimokawabe, and Takayuki Aoki. 2021. GPU Acceleration of Multigrid Preconditioned Conjugate Gradient Solver on Block-Structured Cartesian Grid. In The International Conference on High Performance Computing in Asia-Pacific Region (HPCAsia 2021), January 20-22, 2021, Virtual Event, Republic of Korea. ACM, New York, NY, USA, 9 pages. https: //doi.org/10.1145/3432261.3432273

\section{INTRODUCTION}

In computational science and engineering, it is very important to predict thermal hydraulics based on detailed simulations of the Navier-Stokes equation. Such simulations are also important tools for the safety evaluation in nuclear engineering. We have developed a stencil-based Navier-Stokes solver named JUPITER for predicting three-dimensional multi-phase flows [1]. The JUPITER code can simulate development of gas-liquid interfaces under complex boundary conditions in nuclear reactors. The original JUPITER code is based on a Cartesian grid. However, the use of a Cartesian grid is not so efficient for simulating engineering problems, which contain complicated structures, because a significant overhead is unavoidable from grids assigned to solid structures such as fuel assemblies and regions outside the reactor pressure vessel.

The adaptive mesh refinement (AMR) method is a promising solution to accelerate multi-scale simulations [2]. Since fine grids are arranged only in a region, where smaller scale flows develop, the number of grids can be reduced dramatically, while keeping the accuracy of the simulation. In addition, the block-structured AMR method enables continuous memory access which is essential for efficient computation on state-of-the-art accelerators such as GPUs and many-core processors. We have developed block-AMR CFD 
applications based on the lattice Boltzmann method [3]. However, the lattice Boltzmann method is weakly compressible, and often has accuracy issues in multi-phase flow problems with large density contrasts. Therefore, we develop a block-AMR CFD application based on the Navier-Stokes equation. As a first step, in this work, we redesign the JUPITER code using a block-structured grid with uniform grid resolution, and develop a new Poisson solver on GPUs. This enables us to remove grids in the non-fluid region.

Recently, GPU based simulations have been emerging as an effective technique to accelerate many important classes of scientific applications including CFD applications [4][5]. In the previous study, we have successfully developed a GPU-implementation of JUPITER [6]. The GPU Poisson solvers based on the preconditioned conjugate gradient (P-CG) method and the preconditioned Chebyshev basis communication avoiding method (P-CBCG) [7] achieved good scalability up to 7,680 GPUs on the Summit [6]. However, in the latter work, only the Poisson solver was ported on GPUs, and a full GPU implementation has been the remaining issue.

A peta-scale CFD simulations have a multi-scale feature, and, the number of iterations in Krylov solvers tends to increase with the problem size. The multigrid (MG) method is one of the most promising approaches for this kind of multi-scale problem reaching $\sim 1$ trillion DOFs [8][9]. By improving the convergence property, the number of iterations, and thus, global synchronization can be significantly reduced. However, the remaining issue is to ensure the computational efficiency and the convergence property in the block-structured grid on the many core GPU architecture.

In this study, we develop a full GPU implementation of JUPITER on the block-structured grid. In computing the pressure Poisson equation, a new MG-CG solver is designed to achieve high computational efficiency by fully utilizing the shared memory. We evaluate GPU kernel performance and convergence properties for large-scale two-phase flow problems on the TSUBAME3.0.

\section{RELATED WORK}

GPU implementations of the Poisson solver were discussed in Refs [10][11], and linear algebra libraries such as cuSPARSE [12] and MAGMA [13] are available for GPU computation of small-scale problems.

However, preconditioners require dedicated design and various optimizations for each problem, and it is important to design a tradeoff between the calculation speed and the convergence property. The block Jacobi (BJ) method with the incomplete LU factorization (ILU) is a robust preconditioner, and is often used in CPU computation. The BJ preconditioner on CPUs divides the subdomain on each MPI process into smaller blocks along the continuous memory direction. Each block is then processed by a single OpenMP thread, because ILU in each block cannot be parallelized. However, the concurrency of this algorithm is not enough for GPUs which require several orders of magnitudes larger number of threads. The calculation speed and the convergence property strongly depend on the block size [6]. This requirement becomes even more difficult for the block-structured grid, and thus, it is necessary to develop a new preconditioner.

The MG method is widely used in recent extreme scale simulations [8][9][14]. The most important component of the MG method is a smoother, which satisfies both the computational efficiency and the convergence property. The smoother is classified into local iterative methods such as the Gauss-Seidel method and the Chebyshev iteration method, and global iterative methods such as Krylov subspace methods, which require inner product operations with global reduction communication. Here, the former approaches do not require global reduction communication, and are suitable for massively parallel computing of MG preconditioners. In Ref [14], a communication avoiding $M G$ preconditioner was developed using the Chebyshev iteration smoother with a mixed precision approach, and extreme scale multi-phase CFD simulations with $\sim 90$ billion grids were enabled using $8,000 \mathrm{KNLs}$. However, the concurrency levels required on CPUs and on GPUs are significantly different, and one needs to redesign the whole solver including data structures, algorithms, parallelization, and communication. In this study, we propose a new MG preconditioner, which has high arithmetic intensity using the shared memory and enables continuous memory access on the block-structured grid.

\section{JUPITER CODE}

\subsection{Incompressible Navier-Stokes Solver for Two-Phase Flow}

In this work, we solve two phase flows using an incompressible fluid model based on the Navier-Stokes equation. The Navier-Stokes equation consists of advection, diffusion, and external force terms with an explicit time integration method. Since the divergence-free condition on the velocity field is essential for mass conservation, the pressure Poisson equation is solved iteratively. As a pressurevelocity correction algorithm, the SMAC (Simplified Marker and Cell) method is applied to JUPITER. After solving the pressure Poisson equation, the velocity field is modified with the updated pressure gradient. The time evolutions of the above Navier-Stokes equation are solved as follows:

$$
\begin{gathered}
u_{i}^{*}=u_{i}^{n}+\left(-u_{j}^{n} \frac{\partial u_{i}^{n}}{\partial x_{j}}-\frac{1}{\rho} \frac{\partial p}{\partial x_{i}}+\frac{\partial \tau_{i j}}{\partial x_{j}}\right) \Delta t, \\
\frac{\partial}{\partial x_{i}}\left(\frac{1}{\rho} \frac{\partial \Delta p}{\partial x_{i}}\right)=\frac{1}{\Delta t} \frac{\partial u_{i}^{*}}{\partial x_{i}}, \\
u_{i}^{n+1}=u_{i}^{*}-\frac{1}{\rho} \frac{\partial \Delta p}{\partial x_{i}} \Delta t, \\
p^{n+1}=p^{n}+\Delta p .
\end{gathered}
$$

The dynamics of the gas and liquid phases are described by a convection equation of the volume of fluid $(V O F)$ function, which is based on a phase-field model [15]. A physical property in a cell is determined by the linear weights of the $V O F$ for the gas and liquid phases as follows:

$$
\begin{aligned}
& V O F^{n+1}=V O F^{n}-\frac{\partial u_{j}^{n} V O F^{n}}{\partial x_{j}} \Delta t, \\
& \rho=\rho_{\text {gas }}(1-V O F)+\rho_{\text {liquid }} V O F .
\end{aligned}
$$

Since an interface width of the $V O F$ is kept constant around the grid width by a phase-field model, the density contrast across the gas-liquid interface becomes large with increasing grid resolution, and the numerical stability becomes worse. 


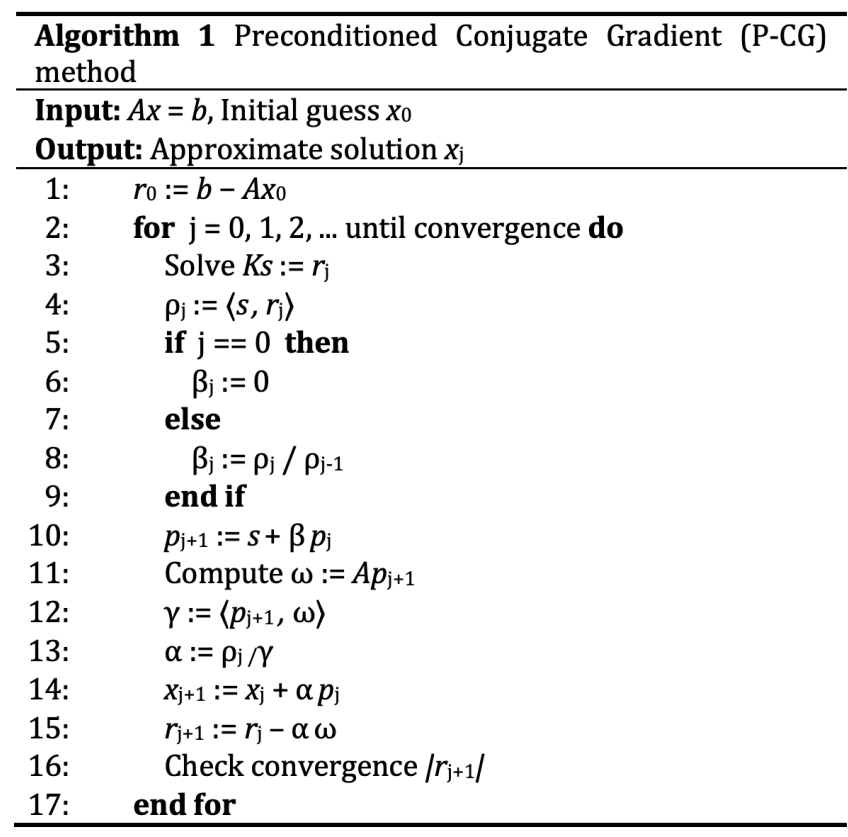

The immersed boundary method (IBM) [16] is applied to the Dirichlet boundary conditions of complex objects. The velocity in the object is modified to satisfy the no-slip condition at the solid-fluid interface. On the other hand, the Neumann boundary condition is applied for the VOF and pressure. Since the gradientfree condition can be completed only in fluid grid next to the solid, no physical quantities inside the solid are referenced or solved in the Poisson solver.

JUPITER has been developed originally in the Cartesian grid. In this work, we re-implement JUPITER in the block-structured grid using CUDA, and develop a new algorithm for the Poisson solver, which occupies a dominant computational cost.

\subsection{Poisson Solver}

The main computational cost comes from the pressure Poisson equation in Eq. 2 which is discretized by the second order accurate centered finite difference scheme with seven stencils. Since the matrix is symmetric and diagonal-dominant, the Poisson solver is normally computed using the P-CG method shown in Algorithm 1. In this work, we use the red-black SOR (RB-SOR) method [17] as a standard preconditioner in the P-CG method. The RB-SOR method has high concurrency and is suitable for GPU computation.

In two-phase flow simulations, a large density ratio between gas and liquid increases the contrast of each matrix component, and this deteriorates the convergence property. Since large-scale simulations have multi-scale features such as thin gas-liquid interfaces and complex object boundaries, the improvement of preconditioning is of critical importance.

\subsection{Geometric Multigrid Preconditioner}

The MG method is one of the most efficient preconditioners to reduce the computational cost and improve the convergence property in multi-scale problems. In the P-CG method in Algorithm 1, we

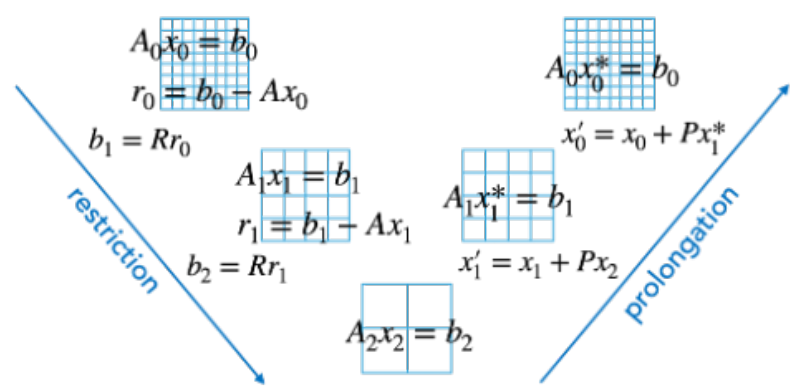

Figure 1: The geometric multigrid preconditioner based on a three-stage V-cycle with fine, middle, and coarse MG levels. The MG levels are defined within each leaf, in which the number of grids is reduced as $8^{3}, 4^{3}$, and $2^{3}$. A fixed iteration number is used for smoothers at each level.

replace the preconditioner in line 3 with a MG preconditioner. Since the JUPITER code is based on the block-structured Cartesian grid system, we can use the geometric multigrid (GMG) method with a three-stage V-cycle shown in Fig. 1.

In the GMG method, an approximate equation $A_{L} x_{L}=b_{L}$ is solved at each MG level $L$. Here $b_{L}$ is the restricted residual from the finer MG level. The interpolation coefficients between MG levels are calculated geometrically. A one-dimensional example is written as follows:

$$
\begin{gathered}
f_{L+1, i}=R f_{L}=f_{L, i-1} / 4+f_{L, i} / 2+f_{L, i+1} / 4, \\
f_{L, i}=P f_{L+1}= \begin{cases}f_{L+1, i}, & (i \in \text { even }) \\
f_{L+1, i} / 2+f_{L+1, i+1} / 2, & (i \in \text { odd })\end{cases}
\end{gathered}
$$

Here $R$ and $P$ are restriction and prolongation operators, respectively. The coefficient of the matrix $A_{L}$ is geometrically obtained from the density on the finer $M G$ level as

$$
\rho_{L+1}=R \rho_{L} \text {. }
$$

The other values on the coarser level are also calculated by the same restriction operator.

The choice of the smoother at each MG level is very important in terms of the computational cost and the convergence property. Although ILU is known as a robust algorithm, it has a data dependency between the calculation grids, and a lot of complicated optimizations are required to obtain reasonable performance on GPUs [6]. The RB-SOR method is an efficient smoother, which has high threads parallelism. However, it requires multiple memory accesses for the red and black phases in order to eliminate the dependency between adjacent grids.

The additive Schwarz method [17] and its multi-color ordering solve this problem. The RB-SOR method is calculated for each local subdomain or leaf, and the leaf data on GPU cache can be reused both in the red and black phases. Our proposed method can effectively reduce the residuals of local subdomain by multiple subiterations.

We use a fixed iteration number for smoothers at each MG level to avoid the convergence check that requires global reduction communication. The number of iterations is chosen based on the initial convergence scan. 


\subsection{Block-Structured Cartesian Grid}

The original JUPITER code was developed based on a Cartesian grid, which can reproduce the shape of the VOF function or the gas-liquid interface with enough accuracy. However, there is a lot of memory wastage for nuclear engineering problems which contain complex non-fluid objects. In the block-structured AMR grid, grid resolution is adaptively changed in each subdomain depending on the local scale of multi-scale solutions [2]. Even without the change of grid resolution, the block-structured grid is beneficial, when the boundary is not aligned to the Cartesian grid and/or complex structures exist inside the computational domain. In this work, we use the block-structured grid with uniform grid resolution and reduce the grids outside the boundary and inside the object.

A data structure is very important from the viewpoint of the computational efficiency in GPU computing. A block-structured grid has an efficient data structure, in which the whole domain is decomposed into block units named "Leaf" [3]. Here, the leaves are connected by the forest-of-octree data structure. Since the leaf consists of a regular Cartesian grid with $n^{3}$ grids, continuous memory access is enabled. Here, larger $n$ improves the efficiency of memory access, while smaller $\mathrm{n}$ leads to better adaptivity to the boundary. In this research, the size of leaf is set to $n=8$ by taking account of the above tradeoff.

The block-structured grid can be easily extended to the MG method. It is possible to use the same forest-of-octree structure as long as there is a grid in the leaf. We adopt the MG method with a three-stage $\mathrm{V}$-cycle, in which the number of grids at fine, middle, and coarse MG levels are set to $8^{3}, 4^{3}$, and $2^{3}$, respectively.

\section{IMPLEMENTATION AND OPTIMIZATION 4.1 Implementation of JUPITER Code on Block-Structured Cartesian Grid}

JUPITER is written in $\mathrm{C}++$ and CUDA. Computational variables are allocated by using the CUDA runtime API "cudaMallocManaged", in which a single pointer is accessible from both CPUs and GPUs. The connection of the block-structured grid is managed by the forest-of-octree data format, in which each variable. Each array is based on the Structure of Array (SoA) memory layout, which is suitable for GPU computing. The same block-structure grid is used also in the MG method, where the offset index changes to $1 / 8$ and $1 / 64$ of the original resolution depending on the MG level.

It is important to assign CUDA threads to grids in stencil calculations. We implement a GPU kernel as shown in Fig. 2. CUDA blocks correspond to the leaves, and CUDA threads are assigned to $8^{3}$ grids in the leaf. Although the block-structured Cartesian grid keeps continuous memory access as in the standard Cartesian grid, special care has to be taken in the treatment of the leaves. Stencil computation for the block-structured grid requires the offset indices of the neighbor leaves, which are normally assigned to the register memory of GPU. This often leads to a lack of the register memory and the resulting performance degradation. As a simple solution to this issue, we use the shared memory to reduce the register usage. In our GPU implementation, CUDA threads are assigned to grids in the leaf. One leaf refers to the offset indices of 26 surrounding leaves, and these indices can be shared by threads

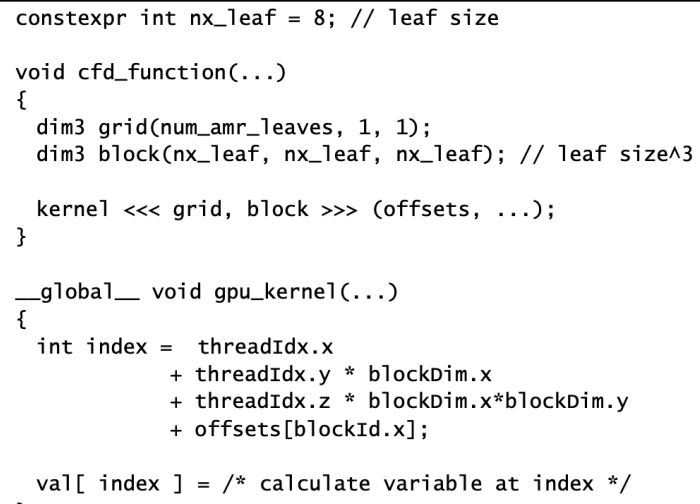

Figure 2: Pseudocode for stencil computation to call GPU instruction.

belonging to the same leaf. Here, the stencil computation refers the neighboring 6 leaves in the $x, y$, and $z$ directions, while the CR-SOR method access 26 surrounding leaves to merge grids at the coarse MG level. We store the indices on the shared memory instead of the register memory. This optimization enables more CUDA threads to be launched simultaneously, and the performance of SpMV and SOR kernels is typically improved by $\sim 50 \%$.

The code is parallelized by using the CUDA-aware MPI library that enables direct communication between GPUs. The computational array is copied to a communication buffer by using a GPU kernel function, and the buffer is transferred by non-blocking pointto-point communication, "MPI Isend" and "MPI Irecv". Each buffer is allocated on the device memory to enable GPU direct RDMA functions.

\subsection{CG Kernel Optimization}

The P-CG method in Algorithm 1 is mainly composed of a vectorvector sum AXPY, a matrix-vector product SpMV (seven stencils), and an inner product of vectors. Since the performances of AXPY and SpMV are limited by memory bandwidth, continuous memory access to the block structured grid improves the efficiency of these kernels.

The performance of inner product is limited by the atomicAdd() function in CUDA. It is important to reduce the total number of reduction operations on global memory. A well-known optimization technique is to use the CUDA Warp Shuffle function for 32 threads in a warp. In this research, we implement further optimization for 1024 threads in a CUDA block by combining the shared memory and the CUDA Warp Shuffle function in Fig.3. Finally, the reduction operation can be implemented using only one atmicAdd() per CUDA block.

\subsection{Red-Black SOR Method}

The RB-SOR method ensures threads parallelism by classifying the entire grids into two colors. CUDA threads can be assigned to $8^{3} / 2,4^{3} / 2$, and $2^{3} / 2$ grids at each $M G$ level, respectively (Fig. 4-(A)). Since GPU computation is performed in units of at least 32 threads of the WARP size on the current NVIDIA GPUs such as P100 


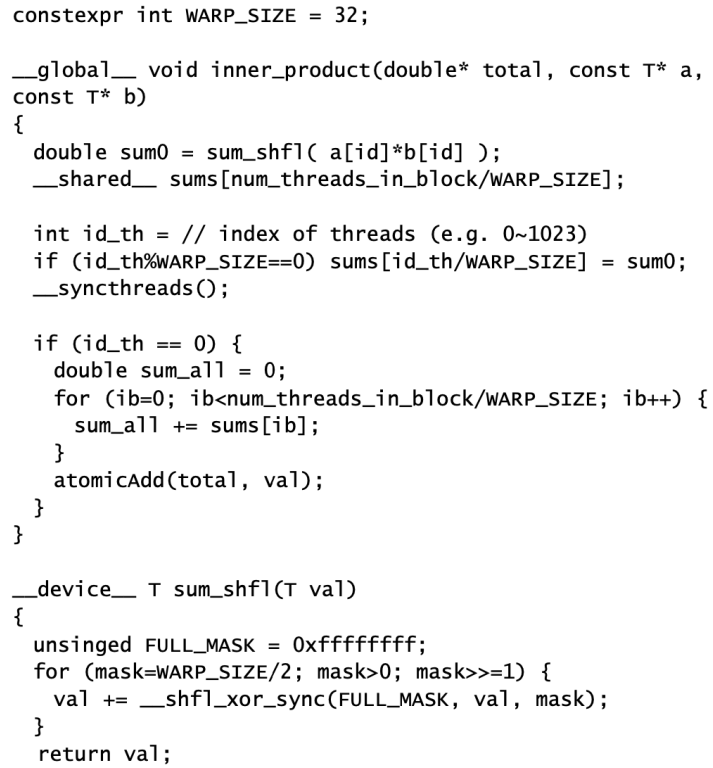

Figure 3: Pseudocode for an inner product of vectors. The first reduction is calculated in the CUDA Warp Shuffle function (sum_shfl) in line 7 . After writing the result to the shared memory in each CUDA Warp (line 10), thread 0 reduces the shared memory (line 16) and call the atomicAdd() function (line 18).

(A)

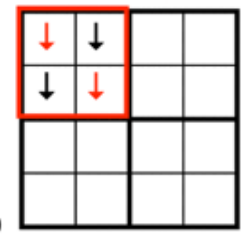

(B)

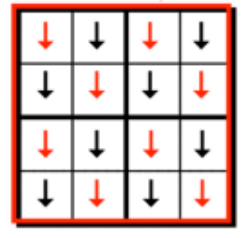

CUDA threads, block

Figure 4: CUDA threads and block configuration of the RBSOR method at the coarse MG level. The leaf is expressed as a thick black box, and the calculation grid is shown by thin black lines. In the standard implementation (A), a single leaf is assigned to a CUDA block. In the optimized implementation (B), eight leaves (in 3D) are computed by a CUDA block.

and V100, a lack of CUDA threads $\left(2^{3} / 2=4\right)$ causes a significant performance degradation at the coarse $M G$ level. To avoid this issue, at the coarse MG level, eight leaves with $2^{3} / 2 \times 8=32$ grids are fused and assigned to one CUDA block as shown in Fig. 4-(B).

\subsection{GPU Cache-Reuse SOR Method}

The calculation speed of smoother is mainly limited by memory $\mathrm{read} /$ write time rather than the cost of floating-point operations. The RB-SOR method requires global memory access twice for the red and black phases, which causes performance degradation. To resolve this issue, we propose a new smoother, which is a variant of the additive Schwarz method [17], consisting of two hierarchical domain decompositions. One is coarse domain decomposition for MPI parallelization (MPI domain), and the other is fine block decomposition for cache optimization (cache block). In the outer iteration, each MPI domain is processed for $\mathrm{n}$ times. Here, cache blocks inside the MPI domain are updated following a two-color ordering. At the fine MG level, halo data communication is processed at every iteration, while at the middle and coarse MG levels, halo data communication is processed only at every two iterations to reduce the communication cost. In the inner iteration, the RB-SOR method within each cache block is computed for $\mathrm{m}$ times to accelerate the convergence. Here, the inner iteration for each cache block is computed on cache, and it has very high arithmetic intensity. However, during the internal iteration, the neighboring cache blocks are not updated.

Figure 5 shows a pseudocode of a cache-reuse optimization version of the RB-SOR (CR-SOR) method. Since the stencil computation on red and black grids are performed within each cache block, the global memory is accessed only once before and after the CR-SOR calculation. NVIDIA Tesla P100 has $64 \mathrm{~KB}$ shared memory per streaming multiprocessor and one CUDA thread block can use 32 $\mathrm{KB}$ each, which can be used as a L1 cache and is shared within a CUDA block. By fully utilizing the high memory bandwidth of the shared memory, the CR-SOR method is enabled with a low computational cost, leading to faster convergence. The CR-SOR method improves both the computational speed and the convergence property compared to the original RB-SOR method.

In this method, we choose different domain decomposition at each MG level. At the fine and middle MG levels, each leaf contains enough grids for GPU computation, and the cache block is chosen to be the same as the leaf, where $(8+2)^{3}$ and $(4+2)^{3}$ grids including halo data are stored on the shared memory, respectively.

On the other hand, at the coarse MG level, we consider different domain decomposition as shown in Fig. 6. Here, each leaf consists of $2^{3}$ grids, which are not enough for GPU computation. To increase the number of grids per cache block, each cache block is defined using $(4+2)^{3}$ grids including halo data, and is located in a staggered manner over $3^{3}$ leaves. This definition cannot cover the whole computational domain, which consists of the forest-of-octree data with even number of leaves in each direction. To cover the whole computational domain, we define eight types of cache blocks, which are centered at even and odd number leaves in each direction, and switch them alternatively in the outer iteration.

Figure 7 shows CUDA threads and block configuration at the coarse MG level. we consider different optimization from the RBSOR method. As shown in Fig. 7-(B), the cache block is defined across $3^{3}=27$ leaves. If one considers a subdomain as in Fig. 4-(B), an extension of halo data requires access to additional 24 leaves in the $\mathrm{x}, \mathrm{y}$, and $\mathrm{z}$ directions, and the total number of leaves per subdomain becomes $8+24=32$. Therefore, the above definition requires access to the minimum number of leaves.

\section{NUMERICAL EXPERIMENT}

The performance of the developed code was measured by simulating two-phase flows in a fuel bundle [19]. The test fluids are water 


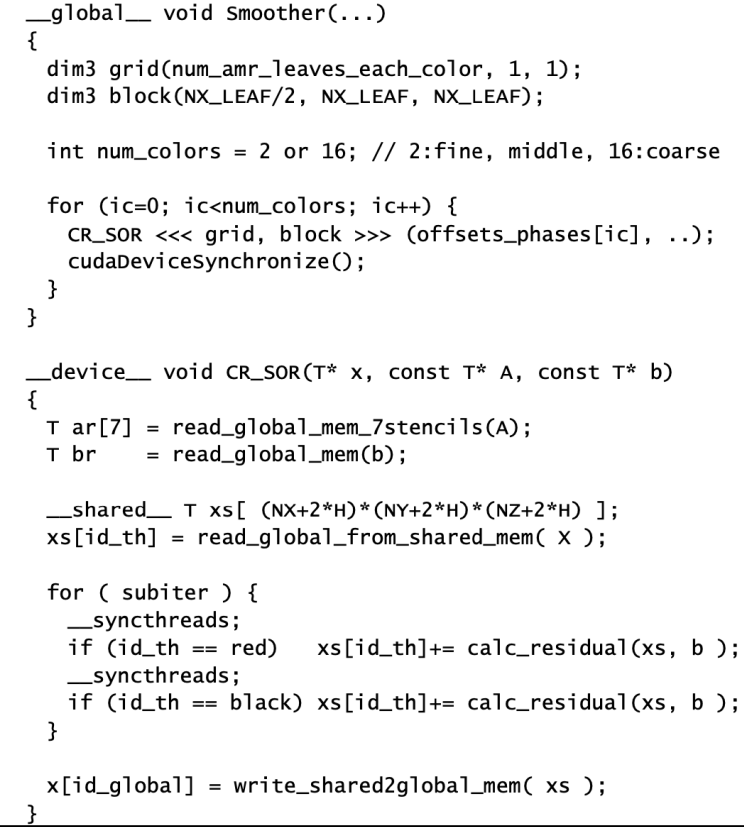

Figure 5: Pseudocode of the cache-reuse (CR) SOR method. The CR-SOR kernel in each subdomain is executed in order following multi-color ordering (line 9). In the RB-SOR kernel, multiple subiterations of red and black phases are calculated on the shared memory (lines 22-26).
(A)

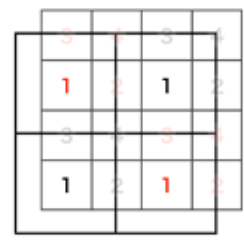

(C)

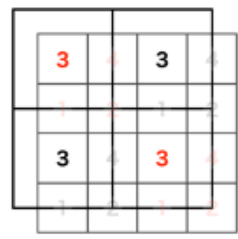

(B)

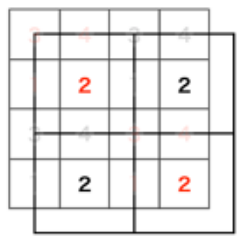

(D)

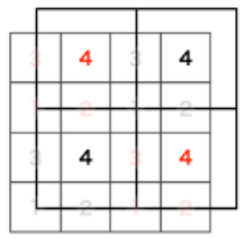

(A)



CUDA threads, block
(B)

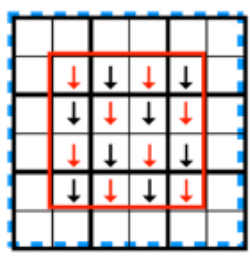

- - - CUDA shared memory
Figure 7: CUDA threads and block configuration of the CRSOR method at the coarse MG level. In the standard implementation (A), a single leaf with a halo region is assigned to a CUDA block. In the optimized implementation (B), 27 leaves (in $3 \mathrm{D}$ ) with a modified halo region are computed by a CUDA block.

Table 1: TSUBAME 3.0 specification of a node.

\begin{tabular}{ccc}
\hline Hardware & Architecture & $\begin{array}{c}\text { Bandwidth } \\
(\mathrm{GB} / \mathrm{s})\end{array}$ \\
\hline CPU & Intel Xeon E5-2680 V4 $(14 \times 2)$ & $153.6(76.8 \times 2)$ \\
GPU & NVIDIA TESLA P100 $\times 4$ & $2928(732 \times 4)$ \\
& Intel Omni-Path HFI $\times 2(\mathrm{CPU})$ & $25(12.5 \times 2)$ \\
Network & Intel Omni-Path HFI $\times 2(\mathrm{GPU})$ & $25(12.5 \times 2)$ \\
\hline \multirow{2}{*}{ Software } & Compiler & Version \\
\hline CPU & GCC & 8.3 .0 \\
GPU & NVIDIA CUDA & 10.2 .89 \\
MPI & OpenMPI & 3.1 .4 \\
\hline
\end{tabular}

an initial condition, bubbles with a radius of $1 \mathrm{~mm}$ were placed between the fuel pins. The grid resolution is $0.101 \mathrm{~mm}$ and the total number of grids correspond to $1,024 \times 1,024 \times 3,072\left(=3.22 \times 10^{9}\right)$ in the Cartesian grid. Since the inside of the fuel pin is solid and does not require CFD computation, the block-structured Cartesian grid can reduce the memory usage without calculation performance degradation. Finally, the total number of grids was reduced to $2.26 \times$ $10^{9}$, which is about $70 \%$ from the original Cartesian grid.

The performance of GPU kernels was measured on the GPUbased supercomputer TSUBAME3.0 shown in Table 1. The compute node consists of two Intel Xeon processors and four NVIDIA TESLA P100 processors. The code is complied with the NVIDIA CUDA Compiler and the OpenMPI.

structured AMR method at the coarse MG level. Small boxes with thin lines denote leaves containing $2^{2}\left(2^{3}\right)$ grids in $2 D$ (3D), and large boxes with bold lines give subdomains in which $3^{2}\left(3^{3}\right)$ leaves are merged. Each subdomain expands to the neighbor leaves, and four and eight types of subdomains are considered in $2 \mathrm{D}$ and $3 \mathrm{D}$ simulation, respectively. The CR-SOR kernel is executed in order from (A) to (D).

and air, and the density ratio is set to 1,000 . Figure 8 shows the computational domain with the size of $104 \mathrm{~mm} \times 104 \mathrm{~mm} \times 312$ $\mathrm{mm}$, in which $8 \times 8$ fuel pins are arranged at equal intervals. As

\subsection{Kernel Performance on NVIDIA Tesla P100}

GPU kernel performance was evaluated against the modified roofline model [4], in which a theoretical processing time of each kernel is estimated by the sum of costs for floating point operations and memory access, $t_{R}=f / F+b / B$. Here, $f$ and $b$ are the number of floating-point operations and memory access of the kernel, and $F$ and $B$ are the peak performance and the memory bandwidth of a single P100 GPU, which has $F=4,700$ GFlops and $B=550$ GBytes/s, where the memory bandwidth is obtained using the Stream benchmark. 
GPU Acceleration of Multigrid Preconditioned Conjugate Gradient Solver on Block-Structured Cartesian Grid

Table 2: Kernel performance analysis on a single GPU: Floating point operation $f$ [Flop/grid], Memory access $b$ [Byte/grid], Arithmetic intensity $f / b$, Peak performance $F$ [Flops], STREAM bandwidth $B$ [Byte/s], Roofline time $t_{R}=f / F+b / B$ [ns/grid], Elapse time $t$ [ns/grid], and Sustained performance $P$ [GFlops]. The CR-SOR kernels are estimated including internal iterations.

\begin{tabular}{ccccccc}
\hline Kernel & $\mathrm{f}$ & $\mathrm{b}$ & $\mathrm{f} / \mathrm{b}$ & $\mathrm{t}$ & $\mathrm{P}$ & $\mathrm{t}_{\mathrm{R}} / \mathrm{t}$ \\
\hline $\begin{array}{c}\text { AXPY } \\
\text { Inner } \\
\text { product } \\
\text { SpMV }\end{array}$ & 3 & 24 & 0.13 & 0.045 & 67.2 & 0.99 \\
\hline $\begin{array}{c}\text { RB-SOR } \\
\text { (Fine) }\end{array}$ & 13 & 72 & 0.18 & 0.141 & 92.2 & 0.95 \\
$\begin{array}{c}\text { RB-SOR } \\
\text { (Middle) }\end{array}$ & 17 & 160 & 0.21 & 0.32 & 53.2 & 0.92 \\
$\begin{array}{c}\text { RB-SOR } \\
\text { (Coarse) }\end{array}$ & 17 & 160 & 0.21 & 0.69 & 24.6 & 0.43 \\
\hline $\begin{array}{c}\text { CR-SOR16 } \\
\text { (Fine) }\end{array}$ & 257 & 80 & 3.21 & 0.45 & 574.6 & 0.48 \\
$\begin{array}{c}\text { CR-SOR8 } \\
\text { (Middle) }\end{array}$ & 129 & 80 & 1.61 & 0.37 & 350.6 & 0.47 \\
$\begin{array}{c}\text { CR-SOR8 } \\
\text { (Coarse) }\end{array}$ & 129 & 80 & 1.61 & 0.47 & 273.0 & 0.37 \\
\hline
\end{tabular}

Table 2 shows the kernel performance analysis for each kernel in the P-CG and MG-CG methods. AXPY and Inner product kernels are memory intensive kernels without neighbor stencil access. Since the block-structured data format enables continuous memory access in the leaf, AXPY kernel achieved ideal sustained performance with the performance ratio against the roofline model $t_{R} / t \sim 0$.99. Inner product kernel also achieved high performance with $t_{R} / t \sim 0.90$ by optimizing the atomic operation. SpMV kernel is very important kernel, because SOR kernels have similar memory access. Thanks to the use of the shared memory, the indices of surrounding leaves can be shared among threads in the leaf, and SpMV kernel achieved high performance with $t_{R} / t \sim 0.95$.

The RB-SOR kernels are processed in the MG preconditioner. Since each kernel accesses global memory twice for the red and black phases, global memory of 10 variables $\times 2$ colors $\times 8=160$ [Byte/grid] is accessed during one iteration in double precision. The performance at the fine and middle MG levels were also high with the roofline ratio of $t_{R} / t \sim 0.92$. At the coarse MG level, the same number of threads as the meddle MG level are generated by combining eight leaves as in Fig. 4-(B), but performance degradation occurred due to fragmentation of memory access. Although the roofline ratio at the coarse MG level becomes a half $t_{R} / t \sim 0.43$, the total calculation time per leaf is only $\sim 4 \%$ compared to the fine MG level.

The CR-SOR method can reduce the memory accesses into a half compared to the RB-SOR method, and the arithmetic intensity is dramatically increased because of rich computation due to internal
HPCAsia 2021, January 20-22, 2021, Virtual Event, Republic of Korea

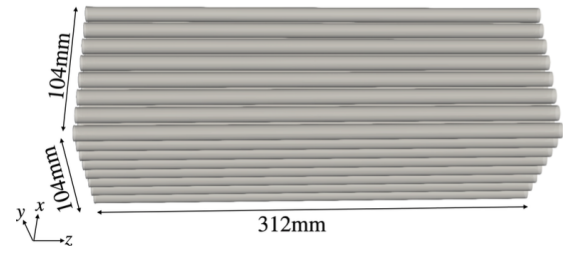

(A)

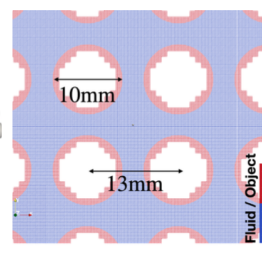

(B)
Figure 8: (A) shows the computational domain for a $8 \times 8$ fuel bundle. The size of the whole domain is $104 \mathrm{~mm} \times 104$ $\mathrm{mm} \times 312 \mathrm{~mm}$. (B) shows the block-structured grid. The diameter of the pin is $10 \mathrm{~mm}$ and the pitch between the fuel pins is $13 \mathrm{~mm}$. Blue and red leaves show fluid and boundary regions, which are allocated in the simulation. Leaves inside the pins (white area) is not allocated.

iterations. The number of internal iterations at the fine, middle, and coarse MG levels is respectively set to 16,8 , and 8 according to the size of subdomain. The CR-SOR method achieved high performance with 574, 350, and 273 GFlops at the fine, middle, and coarse MG levels, respectively. Even with the above internal iterations, the increase of calculation time per grid is less than $\sim 1.5 \times$ from the RB-SOR method.

\subsection{Impact of MG-CG and CRMG-CG Method}

The impacts of the MG-CG methods with the RB-SOR smoother and the CR-SOR smoother (CRMG-CG) are investigated for an $8 \times 8$ fuel bundle in Fig. 8. The whole domain is divided into $4 \times 4 \times 4$ MPI subdomains, and 64 GPUs are assigned to them. Table 3 shows the configuration of the $M G$ preconditioners with a three-stage $\mathrm{V}$-cycle, in which the fine and middle levels are processed twice for restriction and prolongation stages. Here, the RB-SOR and CRSOR methods use the same number of iterations at each MG level, while the latter computes additional internal iterations to accelerate the convergence. The CR-SOR method processes eight types of the coarse subdomains at the coarse MG level. The convergence condition is given by the relative residual error of $|b-A x| / b<10^{-7}$.

Figure 9-(A) shows convergence histories of the P-CG, MG-CG, and CRMG-CG solvers. Although the P-CG solver required more than 1,300 iterations until convergence, the number of iterations in the MG-CG solver was 194, which was less than $15 \%$ of the P-CG solver. The CRMG-SOR solver showed faster convergence with 114 iterations, which was less than $9 \%$ of the P-CG solver.

Figure 9-(B) shows the corresponding computational speeds. It can be confirmed that the calculation speed is dramatically improved by applying the MG-CG and CRMG-CG solvers, which are $\times 3.1$ and $\times 5.9$ faster than the P-CG solver, respectively. These results indicate that the new MG solvers designed for the block-structured data format are effective for large-scale simulations with complex geometries.

\subsection{Strong Scaling Test of CRMG-CG Method}

The strong scaling of JUPITER with the MG-CG and CRMG-CG solvers is summarized in Fig. 10. In the strong scaling test, we use $64,128,192$, and 256 GPUs. In the current strong scaling test, 
Table 3: Configurations of the RB-SOR kernels in the P-CG, MG-CG, and CRMG-CG methods.

\begin{tabular}{ccccc}
\hline Case & MG Lv & $\begin{array}{c}\text { Relaxation } \\
\text { factor }\end{array}$ & $\begin{array}{c}\text { (Outer) } \\
\text { Iterations }\end{array}$ & $\begin{array}{c}\text { Internal } \\
\text { iterations }\end{array}$ \\
\hline $\begin{array}{c}\text { P-CG } \\
\text { RB-SOR }\end{array}$ & Fine & 1.2 & 10 & - \\
\hline MG-CG & & & & \\
RB-SOR & Fine & 1.2 & $\begin{array}{c}5 \times 2 \\
\text { stages }=10\end{array}$ & - \\
RB-SOR & Middle & 1.05 & $\begin{array}{c}25 \times 2 \\
\text { stages }=50\end{array}$ & - \\
RB-SOR & Coarse & 0.99 & 400 & - \\
\hline CRMG-CG & & & & \\
CR-SOR & Fine & 1.2 & $\begin{array}{c}5 \times 2 \\
\text { stages }=10\end{array}$ & 16 \\
CR-SOR & Middle & 1.05 & $\begin{array}{c}25 \times 2 \\
\text { stages }=50\end{array}$ & 8 \\
CR-SOR & Coarse & 0.99 & $\begin{array}{c}50 \times 8 \\
\text { types }=400\end{array}$ & 8 \\
\hline
\end{tabular}

the decomposed domain size is reduced to $1 / 2,1 / 3$, and $1 / 4$ in the $\mathrm{z}$-direction. As the number of GPUs increases, the percentage of the halo leaves increase from $15 \%$ at 64 GPUs to $22 \%$ at 256 GPUs, which causes the performance degradation. Here, the halo leaf means the leaf contains halo grid data, which is referred from the neighboring MPI process. In the current implementation, we perform halo data communication in units of the leaf. The labels of "Precondition", "CG", and "NS" indicate the preconditioner, the CG method including the inner product, and the Navier-Stokes solver with the explicit time integration, respectively.

The breakdown of computational time on 64 GPUs shows that Precondition accounts for more than $90 \%$ of the total time, the remaining cost comes from CG, and NS is negligible. A similar breakdown was observed up to 256 GPUs. In the MG-CG and CRMGCG solvers, global collective communication in the CG method is still negligible, and halo data communication gives a dominant communication cost. The performance gains of the CRMG-CG solver from 64 GPUs are $1.5 \times, 1.9 \times$, and $2.1 \times$ on 128,192 , and 256 GPUs, respectively. It is noted that the calculation speed of the CRMG-CG solver on 256 GPUs is 12 times faster than that of the P-CG solver on 64 GPUs.

\section{SUMMARY AND CONCLUSIONS}

This paper presented a block-structured grid approach and a new MG-CG solver to accelerate a three-dimensional two-phase flow CFD code JUPITER. The MG-CG solver is designed to achieve high performance on GPU-based supercomputers, and the blockstructured grid enables coalesced memory access with keeping the adaptivity of grids to boundaries and objects in the computational domain.

We measured the kernel performance of the P-CG and MG-CG solvers on the GPU based supercomputer TSUBAME 3.0. A treatment of the block-structured data is optimized by storing the offset

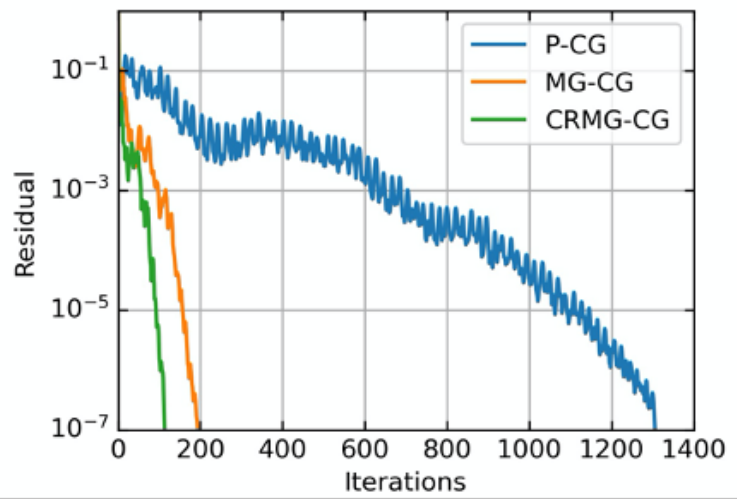

(A)

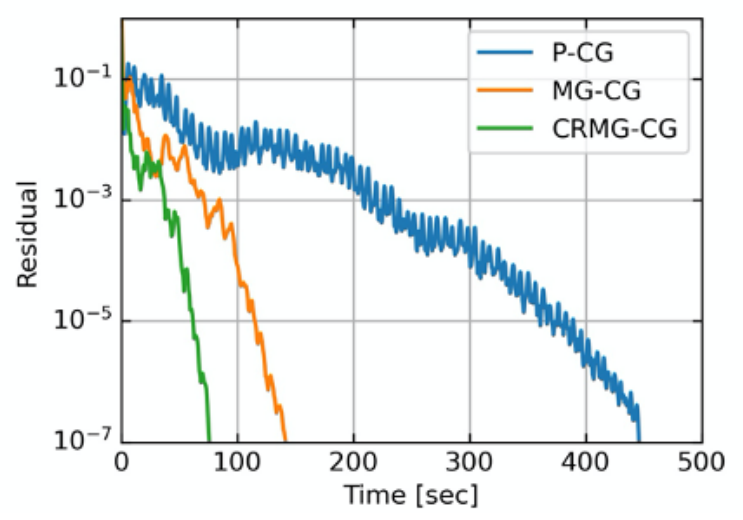

Figure 9: Convergence histories of the P-CG, MG-CG, and CRMG-CG solvers. A large JUPITER matrix from a twophase flow problem in a $8 \times 8$ fuel bundle is computed using 64 GPUs. The P-CG, MG-CG, and CRMG-CG solvers are respectively converged with $1,306,194$, and 114 iterations. The calculation time until convergence of the P-CG, MG-CG, and CRMG-CG solvers are respectively 446, 142, and 76 seconds.

indices of the neighboring leaves on the shared memory. A reduction operation within a CUDA block is highly accelerated by using the shared memory and the CUDA Warp Shuffle function. By these optimization techniques, AXPY, Inner product, and SpMV kernels achieved high performance with $99 \%, 90 \%$, and $95 \%$ of ideal performance in the improved roofline model, respectively.

The performance of the RB-SOR and CR-SOR methods are measured on each MG level. The performance of the RB-SOR at the fine and middle MG levels achieved $90 \%$ of the improved roofline model. At the coarse MG level, we optimized CUDA block assignment, and the calculation time per leaf is suppressed to $\sim 4 \%$ compared to the fine MG level.

The CR-SOR method behaves like direct solvers within a local domain, and successfully reduced global memory access to a half. Thanks to a cache optimization technique by domain decomposition with the additive Schwarz method, multiple internal iterations of the RB-SOR method are efficiently computed on the shared memory. This optimization improves both the arithmetic intensity and the convergence property. The number of internal iterations at the fine, 


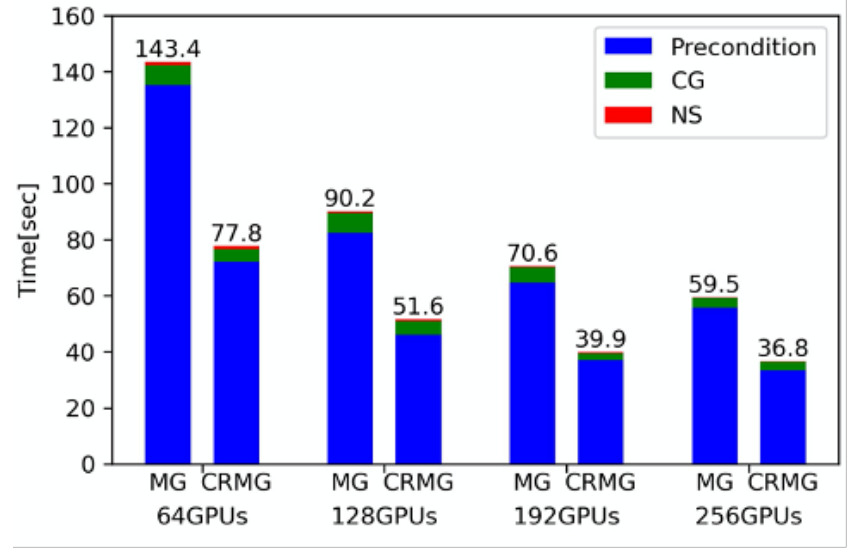

Figure 10: Strong scaling tests of JUPITER with the MG-CG and CRMG-CG solvers up to 256 GPUs. The computational condition is the same as Fig. 9. It is noted that the total time of JUPITER with the P-CG solver is 447.5 second on 64 GPUs.

middle, and coarse MG levels is set to 16,8 , and 8 according to the size of the subdomain, and the CR-SOR method at each MG level achieved high performance of 574, 350, and 273 GFlops, respectively.

We discussed the convergence property under the two-phase flow condition with a fuel bundle. Since our implementation can remove the non-fluid grids, the total number of grids is reduced to $2.26 \times 10^{9}$, which is about $70 \%$ of the original Cartesian grid. The number of iterations of the MG-CG and CRMG-CG methods were less than $15 \%$ and $9 \%$ compared to the P-CG method. It was also confirmed that the calculation speed was dramatically improved, and the MG-CG and CRMG-CG solvers were $\times 3.1$ and $\times 5.9$ faster than the P-CG solver.

The strong scaling of JUPITER with the MG-CG and CRMG-CG solvers were measured from 64 to 256 GPUs. The performance gain of the CRMG-CG solver from 64 GPUs to 256 GPUs was 2.1 times, and the CRMG-CG solver on 256 GPUs was 12 times faster than the P-CG solver on 64 GPUs. We conclude that the redesigned JUPITER code is highly efficient and enables large-scale simulations of two-phase flows on GPU based supercomputers.

\section{ACKNOWLEDGMENTS}

This research was supported in part by the Japan Society for the Promotion of Science (KAKENHI), a Grant-in-Aid for Scientific Research (C) 19K11992 from the Ministry of Education, and Joint Usage/Research Center for Interdisciplinary Large-scale Information Infrastructures in Japan (Project ID: jh190050-NAH, jh200051NAHI). Computations were performed on the TSUBAME at the
Tokyo Institute of Technology, and the ICEX at the Japan Atomic Energy Agency.

\section{REFERENCES}

[1] S. Yamashita, T. Ina, Y. Idomura, and H. Yoshida, "A numerical simulation method for molten material behavior in nuclear reactors," Nucl. Eng. Des., vol. 322, pp. 301-312, 2017.

[2] M. J. Berger and J. Oliger, "Adaptive mesh refinement for hyperbolic partial differential equations," Journal of Computational Physics, vol. 53, no. 3. pp. 484-512, Mar-1984.

[3] N. Onodera, Y. Idomura, Y. Ali, and T. Shimokawabe, "Communication reduced multi-time-step algorithm for real-time wind simulation on GPU-based supercomputers," in Proceedings of ScalA 2018 (Held in conjunction with SC 2018), 2018, pp. 9-16.

[4] T. Shimokawabe et al., "An 80-Fold Speedup, 15.0 TFlops Full GPU Acceleration of Non-Hydrostatic Weather Model ASUCA Production Code," in SC '10: Proceedings of the 2010 ACM/IEEE International Conference for High Performance Computing, Networking, Storage and Analysis, 2010, pp. 1-11.

[5] T. Shimokawabe et al., "Peta-scale phase-field simulation for dendritic solidification on the TSUBAME 2.0 supercomputer,' in SC '11: Proceedings of 2011 International Conference for High Performance Computing, Networking, Storage and Analysis, 2011, pp. 1-11.

[6] Y. Ali, N. Onodera, Y. Idomura, T. Ina, and T. Imamura, "GPU Acceleration of Communication Avoiding Chebyshev Basis Conjugate Gradient Solver for Multiphase CFD Simulations," in Proceedings of ScalA 2019(Held in conjunction with SC 2019), 2019, pp. 1-8.

[7] Y. Kumagai et al., Performance Analysis of the Chebyshev Basis Conjugate Gradient Method on the K Computer. 2016.

[8] T. Ichimura et al., "Implicit nonlinear wave simulation with $1.08 \mathrm{~T}$ DOF and $0.270 \mathrm{~T}$ unstructured finite elements to enhance comprehensive earthquake simulation," in SC '15: Proceedings of the International Conference for High Performance Computing, Networking, Storage and Analysis, 2015, pp. 1-12.

[9] C. Yang et al., 10M-Core Scalable Fully-Implicit Solver for Nonhydrostatic Atmospheric Dynamics. 2016.

[10] I. Yamazaki, S. Rajamanickam, E. G. Boman, M. Hoemmen, M. A. Heroux, and S. Tomov, "Domain Decomposition Preconditioners for Communication-Avoiding Krylov Methods on a Hybrid CPU/GPU Cluster," in The International Conference for High Performance Computing, Networking, Storage and Analysis (SC 14), 2014, pp. 933-944.

[11] K. Matsumoto, Y. Idomura, T. Ina, A. Mayumi, and S. Yamada, "Implementation and performance evaluation of a communication-avoiding GMRES method for stencil-based code on GPU cluster," J. Supercomput., Sep. 2019.

[12] NVIDIA, "cuSPARSE", https://docs.nvidia.com/cuda/cusparse/index.html

[13] Innovative Computing Laboratory, The Univ. Tennessee, "MAGMA", http://icl.cs.utk.edu/magma/

[14] Y. Idomura, T. Ina, S. Yamashita, N. Onodera, S. Yamada, and T. Imamura, "Communication avoiding multigrid preconditioned conjugate gradient method for extreme scale multiphase CFD simulations," in Proceedings of ScalA 2018(Held in conjunction with SC 2018), 2018, pp. 17-24.

[15] P.-H. Chiu and Y.-T. Lin, "A conservative phase field method for solving incompressible two-phase flows," J. Comput. Phys., vol. 230, pp. 185-204, Jan. 2011.

[16] J. Kim, D. Kim, and H. Choi, "An Immersed-Boundary Finite-Volume Method for Simulations of Flow in Complex Geometries," J. Comput. Phys., vol. 171, no. 1, pp. 132-150, 2001.

[17] Y. Saad, Iterative Methods for Sparse Linear Systems. Society for Industrial and Applied Mathematics, 2003.

[18] B. Yang, H. Liu, and Z. Chen, "Preconditioned GMRES solver on multiple-GPU architecture," Comput. Math. with Appl., vol. 72, no. 4, pp. 1076-1095, 2016.

[19] A. ONO, S. YAMASHITA, T. SUZUKI, and H. YOSHIDA, "Numerical simulation of two-phase flow in $4 \times 4$ simulated bundle," Mech. Eng. J., vol. 7, no. 3, pp. 19-583, 2020. 